\title{
IMPROVED UPPER BOUNDS FOR PARTIAL SPREADS
}

\author{
SASCHA KURZ*
}

\begin{abstract}
A partial $(k-1)$-spread in $\mathrm{PG}(n-1, q)$ is a collection of $(k-1)$-dimensional subspaces with trivial intersection such that each point is covered exactly once. So far the maximum size of a partial $(k-1)$-spread in $\mathrm{PG}(n-1, q)$ was know for the cases $n \equiv 0(\bmod k), n \equiv 1(\bmod k)$ and $n \equiv 2(\bmod k)$ with the additional requirements $q=2$ and $k=3$. We completely resolve the case $n \equiv 2(\bmod k)$ for the binary case $q=2$.
\end{abstract}

Keywords: Galois geometry, partial spreads, constant dimension codes, vector space partitions, orthogonal arrays, and $(s, r, \mu)$-nets

MSC: 51E23; 05B15, 05B40, 11T71, 94B25

\section{INTRODUCTION}

For a prime power $q>1$ let $\mathbb{F}_{q}$ be the finite field with $q$ elements and $\mathbb{F}_{q}^{n}$ the standard vector space of dimension $n \geq 1$ over $\mathbb{F}_{q}$. The set of all subspaces of $\mathbb{F}_{q}^{n}$, ordered by the incidence relation $\subseteq$, is called $(n-1)$ dimensional projective geometry over $\mathbb{F}_{q}$ and commonly denoted by $\mathrm{PG}(n-1, q)$. By $G_{q}(n, k)$ we denote the set of all $k$-dimensional subspaces in $\mathrm{PG}(n-1, q)^{1}$. The so-called Gaussian binomial coefficient $\left[\begin{array}{l}n \\ k\end{array}\right]_{q}$, where $\left[\begin{array}{l}n \\ k\end{array}\right]_{q}=\prod_{i=n-k+1}^{n}\left(1-q^{i}\right) / \prod_{i=1}^{k}\left(1-q^{i}\right)$ for $0 \leq k \leq n$ and $\left[\begin{array}{l}n \\ k\end{array}\right]_{q}=0$ otherwise, gives the respective cardinality $\left|G_{q}(n, k)\right|$. A partial $k$-spread in $\mathrm{PG}(n-1, q)$ is a collection of $k$-dimensional subspaces with trivial intersection such that each point ${ }^{2}$, i.e., each element of $G_{q}(n, 1)$, is covered exactly once. A point that is not covered by any of the $k$-dimensional subspaces of the partial $k$-spread is called a hole. We call the number of $k$-dimensional subspaces of a given partial $k$-spread its size and we call it maximum if it has the largest possible size. Bounds for the sizes of maximum partial $k$-spreads were heavily studied in the past. Here we are able to determine the exact value for an infinite series of cases of parameters $n$ and $k$.

Besides the geometric interest in maximum partial $k$-spreads, they also can be seen as a special case of $q$ subspace codes in (network) coding theory. Here the codewords are elements of $\mathrm{PG}(n-1, q)$. Two widely used distance measures for subspace codes (motivated by an information-theoretic analysis of the Koetter-KschischangSilva model, see e.g. [15]) are the so-called subspace distance $d_{S}(U, V):=\operatorname{dim}(U+V)-\operatorname{dim}(U \cap V)=$ $2 \cdot \operatorname{dim}(U+V)-\operatorname{dim}(U)-\operatorname{dim}(V)$ and the so-called injection distance $d_{I}(U, V):=\max \{\operatorname{dim}(U), \operatorname{dim}(V)\}-$ $\operatorname{dim}(U \cap V)$. For $D \subseteq\{0, \ldots, n\}$ we denote by $A_{q}(n, d ; D)$ the maximum cardinality of a subspace code over $\mathbb{F}_{q}^{n}$ with minimum subspace distance at least $d$, where we additionally assume that the dimensions of the codewords are contained in $D$. The most unrestricted case is given by $D=\{0, \ldots, n\}$. The other extreme, $D=\{k\}$ is called constant dimension case and the corresponding codes are called constant dimension codes. As an abbreviation we use the notation $A_{q}(n, d ; k):=A_{q}(n, d ;\{k\})$. Note that $d_{S}(U, V)=2 \cdot d_{I}(U, V) \in 2 \cdot \mathbb{N}$ in the constant dimension case. Bounds on $A_{q}(n, d ; D)$ have been intensively studied in the last years, see e.g. [7]. With this notation, the size of a maximum partial $k$-spread in $\mathbb{F}_{q}^{n}$ is given by $A_{q}(n, 2 k ; k)$.

The remaining part of the paper is structured as follows. We will briefly review some known results on $A_{q}(n, 2 k ; k)$ and discuss their relation with our main result in Section 2 In Section 3 we will provide the technical tools that are then used to prove the main result in Section 4 We close with a conclusion listing some further implications and future lines of research in Section 5

* The work of the author was supported by the ICT COST Action IC1104 and grant KU 2430/3-1 - Integer Linear Programming Models for Subspace Codes and Finite Geometry from the German Research Foundation.

${ }^{1}$ Instead of $\operatorname{PG}(n-1, q)$ we will mainly use the notation $\mathbb{F}_{q}^{n}$ in the following.

${ }^{2}$ In the projective space the dimensions are commonly one less compared to the consideration of subspaces in $\mathbb{F}_{q}^{n}$. 


\section{KNOWN BOUNDS FOR PARTIAL SPREADS}

Counting the points in $\mathbb{F}_{q}^{n}$ and $\mathbb{F}_{q}^{k}$ gives the obvious upper bound $A_{q}(n, 2 k ; k) \leq \frac{\left[\begin{array}{l}n \\ 1\end{array}\right]_{2}}{\left[\begin{array}{c}k \\ 1\end{array}\right]_{2}}=\frac{q^{n}-1}{q^{k}-1}$. If equality is attained one speaks of a $k$-spread.

Theorem 2.1. ([1]; see also [3, p. 29], Result 2.1 in [2]) $\mathbb{F}_{q}^{n}$ contains a $k$-spread if and only if $k$ divides $n$, where we assume $1 \leq k \leq n$ and $k, n \in \mathbb{N}$.

If $k$ does not divide $n$, then we can improve the previous upper bound by rounding down to $A_{q}(n, 2 k ; k) \leq$ $\left\lfloor\frac{q^{n}-1}{q^{k}-1}\right\rfloor$. Here a specific parameterization is useful: If one writes the size of a partial $k$-spread in $\mathbb{F}_{q}^{n}$, where $n=k(t+1)+r, 1 \leq r \leq k-1$, as $q^{r} \cdot \frac{q^{k(t+1)}-1}{q^{k}-1}-s$, then $s \geq q-1$ and $s>\frac{q^{r}-1}{2}-\frac{q^{2 r-k}}{5}$ is known, see e.g. [5]. Furthermore, there exists an example with $s=q^{r}-1$ in each case, see e.g. Observation 3.4 so that there is the conjecture that the sharp bound is $s \geq q^{r}-1$. Assuming $q=2$ and $k \geq 4$, our main result in Theorem 4.3 verifies this conjecture for $r=2$, i.e., $s \geq 3$. Note that $n \equiv r(\bmod k)$, so that the residue class $r$ seems to play a major role. Besides the case of $r=0$, see Theorem 2.1 the next case $r=1$ is solved in full generality:

Theorem 2.2. ([2]; see also [14] for the special case $q=2$ ) For positive integers $1 \leq k \leq n$ be positive integers with $n \equiv 1(\bmod k)$ we have $A_{q}(n, 2 k ; k)=\frac{q^{n}-q}{q^{k}-1}-q+1=q \cdot \frac{q^{n-1}-1}{q^{k}-1}-q+1=\frac{q^{n}-q^{k+1}+q^{k}-1}{q^{k}-1}$.

The so far best upper bound on $A_{q}(n, 2 k ; k)$, i.e., the best known lower bound on $s$ is based on:

Theorem 2.3. (Corollary 8 in [4]) If $n=k(t+1)+r$ with $0<r<k$, then

$$
A_{q}(n, 2 k ; k) \leq \sum_{i=0}^{t} q^{i k+r}-\lfloor\theta\rfloor-1=q^{r} \cdot \frac{q^{k(t+1)}-1}{q^{k}-1}-\lfloor\theta\rfloor-1,
$$

where $2 \theta=\sqrt{1+4 q^{k}\left(q^{k}-q^{r}\right)}-\left(2 q^{k}-2 q^{r}+1\right)$.

We remark that this theorem is also restated as Theorem 13 in [7] and as Theorem 44 in [9] with the small typo of not rounding down $\theta$ ( $\Omega$ in their notation). And indeed, the resulting lower bound $s \geq\lfloor\theta(q, k, r)\rfloor+1$ is independent of $n$. Specializing to the binary case, i.e., $q=2$, we can use the previous results to state exact formulas for $A_{2}(n, 2 k ; 2 k)$ for small values of $k \geq 22^{3}$

From Theorem 2.1 and Theorem 2.2 we conclude:

Corollary 2.4. For each integer $m \geq 2$ we have

(a) $A_{2}(2 m, 4 ; 2)=\frac{2^{2 m}-1}{3}$;

(b) $A_{2}(2 m+1,4 ; 2)=\frac{2^{2 m+1}-5}{3}$.

Using the results of Theorem 2.1 and Theorem 2.2 the case $k=3$ was completely settled in [6]:

Theorem 2.5. For each integer $m \geq 2$ we have

(a) $A_{2}(3 m, 6 ; 3)=\frac{2^{3 m}-1}{7}$;

(b) $A_{2}(3 m+1,6 ; 3)=\frac{2^{3 m+1}-9}{7}$;

(c) $A_{2}(3 m+2,6 ; 3)=\frac{2^{3 m+2}-18}{7}$.

In our Theorem 4.3 we completely settle the case $n \equiv 2(\bmod k)$ for $q=2, k \geq 4$, and $n \geq 2 k+2 \bigsqcup^{4}$ Using the results of Theorem 2.1, Theorem 2.2, Observation 3.4 and Theorem 4.3 we can state:

Corollary 2.6. For each integer $m \geq 2$ we have

(a) $A_{2}(4 m, 8 ; 4)=\frac{2^{4 m}-1}{15}$;

(b) $A_{2}(4 m+1,8 ; 4)=\frac{2^{4 m+1}-17}{15}$;

(c) $A_{2}(4 m+2,8 ; 4)=\frac{2^{4 m+2}-49}{15}$;

\footnotetext{
${ }^{3}$ Obviously, we have $A_{q}(n, 2 ; 1)=\left[\begin{array}{c}n \\ 1\end{array}\right]_{q}$.

${ }^{4}$ As $A_{q}(k+2,2 k ; k)=1$ for $k \geq 2$, the assumption $n \geq 2 k+2$ is no restriction. The case $k=3$ is covered by [6], see Theorem 2.5 For $k=1,2$ the remainder of $n$ is strictly smaller than 2 . So, in other words, the binary case $n \equiv 2(\bmod k)$ is completely resolved.
} 
(d) $\frac{2^{4 m+3}-113}{15} \leq A_{2}(4 m+3,8 ; 4) \leq \frac{2^{4 m+3}-53}{15}$.

In [7] Etzion collects 100 open problems on $q$-analogs in coding theory. Our main theorem resolves several of them:

- In other words, Research problem 45 asks for a characterization of parameter cases for which the construction in Observation 3.4 matches the exact value of $A_{q}(n, 2 k ; k)$. Assuming $q=2$ and $k \geq 4$, this is, e.g., the case for $n \equiv 2(\bmod k)$.

- Research problem 46 asks for improvements of the upper bound from Theorem 2.3 which are achieved for the same parameters as specified above. The same is true for Research problem 47 asking for exact values.

- The special case of the determination of $A_{2}(n, 8 ; 4)$ in Research problem 49 is completely resolved for $n \equiv 2(\bmod 4)$, see Corollary 2.6 .

\section{CONSTRUCTIONS AND VECTOR SPACE PARTITIONS}

For matrices $A, B \in \mathbb{F}_{q}^{m \times n}$ the rank distance is defined via $d_{R}(A, B):=\operatorname{rk}(A-B)$. It is indeed a metric, as observed in [10].

Theorem 3.1. (see [10]) Let $m, n \geq d$ be positive integers, $q$ a prime power, and $\mathcal{C} \subseteq \mathbb{F}_{q}^{m \times n}$ be a rank-metric code with minimum rank distance $d$. Then, $|\mathcal{C}| \leq q^{\max (n, m) \cdot(\min (n, m)-d+1)}$. Codes attaining this upper bound are called maximum rank distance (MRD) codes. They exist for all (suitable) choices of parameters.

If $m<d$ or $n<d$, then only $|\mathcal{C}|=1$ is possible, which may be summarized to the single upper bound $|\mathcal{C}| \leq\left\lceil q^{\max (n, m) \cdot(\min (n, m)-d+1)}\right\rceil$. Using an $m \times m$ identity matrix as a prefix one obtains the so-called lifted MRD codes.

Theorem 3.2. (see [15]) For positive integers $k, d, n$ with $k \leq n, d \leq 2 \min (k, n-k)$, and $d \equiv 0(\bmod 2)$, the size of a lifted MRD code in $G_{q}(n, k)$ with subspace distance $d$ is given by

$$
M(q, k, n, d):=q^{\max (k, n-k) \cdot(\min (k, n-k)-d / 2+1)} .
$$

If $d>2 \min (k, n-k)$, then we have $M(q, k, n, d)=1$.

In [8] a generalization, the so-called multi-level construction, was presented. To this end let $1 \leq k \leq n$ be integers and $v \in \mathbb{F}_{2}^{n}$ a binary vector of weight $k$. By $\mathrm{EF}(v)$ we denote the set of all $k \times n$ matrices over $\mathbb{F}_{2}$ that are in row-reduced echelon form, i.e., the Gaussian algorithm had been applied, and the pivot columns coincide with the positions where $v$ has a 1-entry.

Theorem 3.3. (see [8]) For integers $k, n, d$ with $1 \leq k \leq n$ and $1 \leq d \leq \min (k, n-k)$, let $\mathcal{B}$ be a binary constant weight code of length $n$, weight $k$, and minimum Hamming distance $2 d$. For each $b \in \mathcal{B}$ let $\mathcal{C}_{b}$ be $a$ code in $\mathrm{EF}(b)$ with minimum rank distance at least $d$. Then, $\cup_{b \in \mathcal{B}} \mathcal{C}_{b}$ is a constant dimension code of dimension $k$ having a subspace distance of at least $2 d$.

The authors also came up with a conjecture for the size of an MRD code over $\mathrm{EF}(v)$, which is still unrebutted. Taking binary vectors with $k$ consecutive ones we are in the classical MRD case. So, taking binary vectors $v_{i}$, where the ones are located in positions $(i-1) k+1$ to $i k$, for all $1 \leq i \leq\lfloor n / k\rfloor$, clearly gives a binary constant weight code of length $n$, weight $k$, and minimum Hamming distance $2 k$.

Observation 3.4. For positive integers $k, n$ with $n>2 k$ and $n \not \equiv 0(\bmod k)$, there exists a constant dimension code in $G_{q}(n, k)$ with subspace distance $2 k$ having cardinality

$$
1+\sum_{i=1}^{\lfloor n / k\rfloor-1} q^{n-i k}=1+q^{k+(n \bmod k)} \cdot \frac{q^{n-k-(n \bmod k)}-1}{q^{k}-1}=\frac{q^{n}-q^{k+(n \bmod k)}+q^{k}-1}{q^{k}-1}
$$

\footnotetext{
${ }^{5}$ Using our general notation, we may rewrite the stated formula with $n=k(t+1)+r$ and $n \bmod k=r$.
} 
We remark that a more general construction, among similar lines and including explicit formulas for the respective cardinalities, has been presented in [16].

A vector space partition $\mathcal{P}$ of $\mathbb{F}_{q}^{n}$ is a collection of subspaces with the property that every nonzero vector, i.e., a point, of $\mathbb{F}_{q}^{n}$ is contained in a unique member of $\mathcal{P}$. If for $d \in\{1,2, \ldots, k\}$ the vector space partition $\mathcal{P}$ contains $m_{d}$ subspaces of dimension $d$ and $m_{k}>0$, then $\left(m_{k}, m_{k-1}, \ldots, m_{1}\right)$ is called the type of $\mathcal{P}$. We will also use the notation $k^{m_{k}} \ldots 1^{m_{i}}$, where we leave out the cases with $m_{d}=0$. The tail of $\mathcal{P}$ is the set of subspaces, in $\mathcal{P}$, having the smallest dimension. If the dimension of the corresponding subspaces is given by $d$, then the length of the tail is the number $m_{d}$, i.e., the cardinality of the tail.

Theorem 3.5. (Theorem 1 in [11]) Let $\mathcal{P}$ be a vector space partition of $\mathbb{F}_{q}^{n}$, let $n_{1}$ denote the length of the tail of $\mathcal{P}$, let $d_{1}$ denote the dimension of the vector spaces in the tail of $\mathcal{P}$, and let $d_{2}$ denote the dimension of the vector spaces of the second lowest dimension.

(i) if $q^{d_{2}-d_{1}}$ does not divide $n_{1}$ and if $d_{2}<2 d_{1}$, then $n_{1} \geq q^{d_{1}}+1$;

(ii) if $q^{d_{2}-d_{1}}$ does not divide $n_{1}$ and if $d_{2} \geq 2 d_{1}$, then either $d_{1}$ divides $d_{2}$ and $n_{1}=\left(q^{d_{2}}-1\right) /\left(q^{d_{1}}-1\right)$ or $n_{1}>2 q^{d_{2}-d_{1}}$

(iii) if $q^{d_{2}-d_{1}}$ divides $n_{1}$ and $d_{2}<2 d_{1}$, then $n_{1} \geq q^{d_{2}}-q^{d_{1}}+q^{d_{2}-d_{1}}$;

(iv) if $q^{d_{2}-d_{1}}$ divides $n_{1}$ and $d_{2} \geq 2 d_{1}$, then $n_{1} \geq q^{d_{2}}$.

So, in any (nontrivial) case $e^{6}$, we have $n_{1} \geq q+1 \geq 3$, which will be sufficient in many situations.

\section{MAIN THEOREM}

Lemma 4.1. For two integers $t \geq 1$ and $k \geq 4$ no vector space partition of type $k^{n_{k}}(k-1)^{n_{k-1}} 1^{1+2^{k-1}}$ exists in $\mathbb{F}_{2}^{k(t+1)+1}$, where $n_{k}=\frac{2^{k t+2}+2^{k}-5}{2^{k}-1}$ and $n_{k-1}=2^{k t+2}-3.7$

PROOF. Assume the existence of a vector space partition $\mathcal{P}$ of the specified type. Now we consider the projection into hyperplanes. Since the non-holes are projected to $m=\frac{2^{k(t+1)+2}-2^{k+1}-2}{2^{k}-1}$ subspaces with dimensions in $\{k, k-1, k-2\}$ and the total number of points is given by $\left[\begin{array}{c}k(t+1) \\ 1\end{array}\right]_{2}=2^{k(t+1)}-1$, the number of holes $L$ has to satisfy $L \equiv 1\left(\bmod 2^{k-2}\right)$. Using $L \leq 1+2^{k-1}$, we conclude $L \in\left\{1,1+2^{k-2}, 1+2^{k-1}\right\}$. Due to the tail condition in Theorem 3.5 , the case $L=1$ is impossible. Now let $x$ be the number of hyperplanes with $1+2^{k-1}$ holes and $\left[\begin{array}{c}k(t+1)+1 \\ k(t+1)\end{array}\right]_{2}-x=2^{k(t+1)+1}-1-x$ the number of hyperplanes with $1+2^{k-2}$ holes. Since each hole is contained in $\left[\begin{array}{c}k(t+1) \\ k(t+1)-1\end{array}\right]_{2}=2^{k(t+1)}-1$ hyperplanes, we have

$$
\begin{aligned}
\frac{\left(1+2^{k-1}\right) x+\left(1+2^{k-2}\right) \cdot\left(2^{k(t+1)+1}-1-x\right)}{2^{k(t+1)}-1} & =\frac{\left(1+2^{k-2}\right) \cdot 2^{k(t+1)+1}-\left(1+2^{k-2}\right)+2^{k-2} \cdot x}{2^{k(t+1)}-1} \\
& \geq \frac{\left(1+2^{k-2}\right) \cdot 2^{k(t+1)+1}-\left(1+2^{k-2}\right)}{2^{k(t+1)}-1} \\
& >2 \cdot\left(1+2^{k-2}\right)=2^{k-1}+2
\end{aligned}
$$

holes in total, a contradiction.

Lemma 4.2. Using the notation from Theorem 2.3 we have $\lfloor\theta\rfloor=\left\lfloor\frac{q^{r}-2}{2}\right\rfloor$ for $r \geq 1$ and $\left.k \geq 2 r\right]^{8}$

ProOF. We have

$$
2 \theta=\sqrt{1+4 q^{k}\left(q^{k}-q^{r}\right)}-\left(2 q^{k}-2 q^{r}+1\right)=\sqrt{\left(2 q^{k}-q^{r}\right)^{2}-q^{2 r}+1}-\left(2 q^{k}-2 q^{r}+1\right)<q^{r}-1 .
$$

Since $1+4 q^{k}\left(q^{k}-q^{r}\right)=1+4 q^{2 k}-4 q^{k+r}>\left(2 q^{k}-\left(q^{r}+1\right)\right)^{2}=4 q^{2 k}-4 q^{k+r}-4 q^{k}+q^{2 r}+2 q^{r}+1$ for $k \geq 2 r$ and $q \geq 2$, we have $2 \theta>q^{r}-2$. Thus, we have $\lfloor\theta\rfloor=\left(q^{r}-2\right) / 2$ for $q$ even and $\lfloor\theta\rfloor=\left(q^{r}-3\right) / 2$ for $q$ odd.

\footnotetext{
${ }^{6}$ We have to exclude the trivial subspace partition $\mathcal{P}=\left\{\mathbb{F}_{q}^{n}\right\}$, where $d_{1}=n$ and $d_{2}$ does not exist.

${ }^{7}$ Theorem 3.5 (ii,iv) yields $n_{1} \geq 2^{k-1}-1$, setting $d_{2}=k-1$ and $d_{1}=1$. Also the improvement [12] Theorem 2] of Theorem 3.5 is not sufficient to exclude the specific parameters from Lemma 4.1

${ }^{8}$ The result is also valid for $k=2 r-1, r \geq 2$, and $q \in\{2,3\}$.
} 
We remark that the formula for $\lfloor\theta\rfloor$ in Lemma 4.2 does not depended on $k$ (supposed that $k$ is sufficiently large).

Theorem 4.3. For each pair of integers $t \geq 1$ and $k \geq 4$ we have $A_{2}(k(t+1)+2,2 k ; k)=\frac{2^{k(t+1)+2}-3 \cdot 2^{k}-1}{2^{k}-1}$. Proof. Applying Lemma 4.2 and Theorem 2.3 yields $A_{2}(k(t+1)+2,2 k ; k) \leq \frac{2^{k(t+1)+2}-2^{k+1}-2}{2^{k}-1}$. Assuming that the upper bound $m:=\frac{2^{k(t+1)+2}-2^{k+1}-2}{2^{k}-1}$ is attained by a code $\mathcal{C}$, we obtain a vector space partition of type $k^{m} 1^{2^{k+1}+1}$, i.e., the $m k$-dimensional codewords leave over $\left[\begin{array}{c}k(t+1)+2 \\ 1\end{array}\right]_{2}-m \cdot\left[\begin{array}{l}k \\ 1\end{array}\right]_{2}=2^{k(t+1)+2}-1-$ $\frac{2^{k(t+1)+2}-2^{k+1}-2}{2^{k}-1} \cdot\left(2^{k}-1\right)=2^{k+1}+1$ holes. Now we consider the projection of $\mathcal{C}$ into hyperplanes. Since the codewords end up as $k$ - or $(k-1)$-dimensional subspaces summing up to $m$, the number of holes is at most $2^{k+1}+1$, and the total number of points is given by $\left[\begin{array}{c}k(t+1)+1 \\ 1\end{array}\right]_{2}=2^{k(t+1)+1}-1$, we obtain the following list of possible types:

(1) $k^{n_{k}+1}(k-1)^{n_{k-1}-1} 1^{1}$

(2) $k^{n_{k}}(k-1)^{n_{k-1}} 1^{1+2^{k-1}}$

(3) $k^{n_{k}-1}(k-1)^{n_{k-1}+1} 1^{1+2^{k}}$

(4) $k^{n_{k}-2}(k-1)^{n_{k-1}+2} 1^{1+3 \cdot 2^{k-1}}$

(5) $k^{n_{k}-3}(k-1)^{n_{k-1}+3} 1^{1+2^{k+1}}$,

where $n_{k}=\frac{2^{k t+2}+2^{k}-5}{2^{k}-1}$ and $n_{k-1}=2^{k t+2}-3$.

Due to Theorem 3.5, case (1) is impossible. The case (2) is ruled out by Lemma 4.1 Thus, each of the $\left[\begin{array}{l}k(t+1)+2 \\ k(t+1)+1\end{array}\right]_{2}=2^{k(t+1)+2}-1$ hyperplanes contains at most $n_{k}-1$ subspaces of dimension $k$. Since each $k$-dimensional subspace is contained in $\left[\begin{array}{l}k t+2 \\ k t+1\end{array}\right]_{2}=2^{k t+2}-1$ hyperplanes, the total number of $k$-dimensional subspaces in $\mathcal{C}$ can be at most

$$
\frac{\left(2^{k(t+1)+2}-1\right) \cdot\left(n_{k}-1\right)}{2^{k t+2}-1}=\frac{2^{k(t+1)+2}-1}{2^{k}-1}-3 \cdot \frac{2^{k(t+1)+2}-1}{\left(2^{k}-1\right) \cdot\left(2^{k t+2}-1\right)} \stackrel{k>0}{<} \frac{2^{k(t+1)+2}-3 \cdot 2^{k}-1}{2^{k}-1},
$$

a contradiction. Thus we have $A_{2}(k(t+1)+2,2 k ; k) \leq \frac{2^{k(t+1)+2}-3 \cdot 2^{k}-1}{2^{k}-1}$. A construction for $A_{2}(k(t+1)+$ $2,2 k ; k) \geq \frac{2^{k(t+1)+2}-3 \cdot 2^{k}-1}{2^{k}-1}$ is given by Observation 3.4

Corollary 4.4. For each integer $k \geq 4$ we have $A_{2}(2 k+2,2 k ; k)=2^{k+2}+1$.

We remark that Corollary 4.4 would be wrong for $k=3$, since $A_{2}(8,6 ; 3)=34>33$, see [6]. And indeed, each extremal code has to contain a hyperplane which is a subspace partition of type $3^{5} 2^{29} 1^{5}$. Next we try to get a bit more information about these extremal codes. To this end let $a_{i}$ denote the number of hyperplanes containing exactly $2 \leq i \leq 5$ three-dimensional codewords. The standard equations for our parameters are given by

$$
\begin{aligned}
a_{2}+a_{3}+a_{4}+a_{5} & =\left[\begin{array}{l}
8 \\
7
\end{array}\right]_{2}=255 \\
2 a_{2}+3 a_{3}+4 a_{4}+5 a_{5} & =\left[\begin{array}{l}
5 \\
1
\end{array}\right]_{2} \cdot A_{2}(8,6 ; 3)=1054 \\
a_{2}+3 a_{3}+6 a_{4}+10 a_{5} & =\left(\begin{array}{c}
A_{2}(8,6 ; 3) \\
2
\end{array}\right)=561 .
\end{aligned}
$$

Solving the equation system for $a_{5}$ yields $a_{4}=7 a_{5}-782, a_{3}=2108-17 a_{5}$, and $a_{2}=9 a_{5}-1071$. Since the $a_{i}$ have to be non-negative we obtain $119 \leq a_{5} \leq 124$, i.e., the list of theoretically possible spectra is given by $(0,0,0,85,51,119),(0,0,9,68,58,120),(0,0,18,51,65,121),(0,0,27,34,72,122),(0,0,36,17,79,123)$, and $(0,0,45,0,86,124)$.

Now let $L$ be the subspace generated by the 17 holes. Since $17>15$ we have $\operatorname{dim}(L) \in\{5,6,7,8\}$. The hyperplane containing 2 codewords contains all 17 holes so that the set of hyperplanes of this type corresponds to the set of hyperplanes containing $L$ as a subspace, i.e., $\operatorname{dim}(L)=8-i$ is equivalent to $a_{2}=2^{i}-1$ for $1 \leq i \leq 3$. Thus, we have $\operatorname{dim}(L)=8$ and the unique spectrum is given by $(0,0,0,85,51,119)$, i.e., $a_{2}=0$, $a_{3}=85, a_{4}=51$, and $a_{5}=119$. 
We remark that Lemma 4.1 can be generalized to arbitrary odd 9 prime powers $q$ along the same lines:

Lemma 4.5. For integers $t \geq 1, k \geq 4$, and odd $q$ no vector space partition of type $k^{p-1}(k-1)^{m-p+1} 1^{\frac{q+1}{2}+q^{k-1}}$ exists in $\mathbb{F}_{q}^{k(t+1)+1}$, where $p=\frac{q^{k t+2}-q^{2}}{q^{k}-1}+\frac{q+1}{2}$ and $m=\frac{q^{k(t+1)+2}-q^{2}}{q^{k}-1}-\frac{q^{2}-1}{2}$.

PROOF. Assume the existence of a vector space partition $\mathcal{P}$ of the specified type. Now we consider the projection into hyperplanes. Since the non-holes are projected to $m$ subspaces with dimensions in $\{k, k-1, k-2\}$ and the total number of points is given by $\left[\begin{array}{c}k(t+1) \\ 1\end{array}\right]_{q}$, the number of holes $L$ has to satisfy $L \equiv \frac{q+1}{2}\left(\bmod q^{k-2}\right)$. Using $L \leq \frac{q+1}{2}+q^{k-1}$, we conclude $L \in\left\{\frac{q+1}{2}, \frac{q+1}{2}+q^{k-2}, \frac{q+1}{2}+q^{k-1}\right\}$. Due to the tail condition in Theorem 3.5 , the case $L=\frac{q+1}{2}$ is impossible. Now let $x$ be the number of hyperplanes with $\frac{q+1}{2}+q^{k-1}$ holes and $\left[\begin{array}{c}k(t+1)+1 \\ k(t+1)\end{array}\right]_{q}-x$ the number of hyperplanes with $\frac{q+1}{2}+q^{k-2}$ holes. Since each hole is contained in $\left[\begin{array}{c}k(t+1) \\ k(t+1)-1\end{array}\right]_{q}$ hyperplanes, we have

$$
\begin{aligned}
& \frac{\left(\frac{q+1}{2}+q^{k-1}\right) x+\left(\frac{q+1}{2}+q^{k-2}\right) \cdot\left(\frac{q^{k(t+1)+1}-1}{q-1}-x\right)}{\left(q^{k(t+1)}-1\right) /(q-1)} \\
= & q^{k-2} x+\left(\frac{q+1}{2}+q^{k-2}\right) \cdot \frac{q^{k(t+1)+1}-1}{q^{k(t+1)}-1} \\
\geq & \left(\frac{q+1}{2}+q^{k-2}\right) \cdot q>\frac{q+1}{2}+q^{k-1}
\end{aligned}
$$

holes in total, a contradiction.

In turns out that repeating the proof of Theorem 4.3 for odd $q$ just works for $q=3$ and additionally the lower bound by the constriction of Observation 3.4 does not match the improved upper bound. At the very least an improvement of the upper bound of Theorem 2.3 by one is possible:

Lemma 4.6. For integers $t \geq 1$ and $k \geq 4$ we have $A_{3}(k(t+1)+2,2 k ; k) \leq \frac{3^{k(t+1)+2}-3^{2}}{3^{k}-1}-\frac{3^{2}+1}{2}$.

PROOF. Applying Lemma 4.2 and Theorem 2.3 for odd $q$ yields

$$
A_{q}(k(t+1)+2,2 k ; k) \leq \frac{q^{k(t+1)+2}-q^{2}}{q^{k}-1}-\frac{q^{2}-1}{2}=: m .
$$

Assuming that the upper bound is attained by a code $\mathcal{C}$, the $m k$-dimensional codewords leave over

$$
\left[\begin{array}{c}
k(t+1)+2 \\
1
\end{array}\right]_{q}-m \cdot\left[\begin{array}{l}
k \\
1
\end{array}\right]_{q}=\frac{q(q+1)}{2} \cdot q^{k-1}+\frac{q+1}{2}=: h
$$

holes. Now we consider the projection of $\mathcal{C}$ into hyperplanes. Since the codewords end up as $k$ - or $(k-1)$ dimensional subspaces summing up to $m$, the number of holes is at most $h$, and the total number of points is given by $\left[\begin{array}{c}k(t+1)+1 \\ 1\end{array}\right]_{q}=\frac{q^{k(t+1)+1}-1}{q-1}$, we obtain the types

$$
k^{p-i}(k-1)^{m-p+i} 1^{\frac{q+1}{2}+i q^{k-1}}
$$

for $0 \leq i \leq \frac{q(q+1)}{2}$, where $p:=\frac{q^{k t+2}-q^{2}}{q^{k}-1}+\frac{q+1}{2}$.

Due to Theorem 3.5. case $i=0$ is impossible. The case $i=1$ is ruled out by Lemma 4.5 Thus, each of the $\left[\begin{array}{c}k(t+1)+2 \\ k(t+1)+1\end{array}\right]_{q}$ hyperplanes contains at most $p-2$ subspaces of dimension $k$. Since each $k$-dimensional subspace

\footnotetext{
${ }^{9}$ For even $q>2$ the tail condition of Theorem 3.5 cannot be applied directly in the proof of Lemma 4.5
} 
is contained in $\left[\begin{array}{l}k t+2 \\ k t+1\end{array}\right]_{q}$ hyperplanes, the total number of $k$-dimensional subspaces in $\mathcal{C}$ can be at most

$$
\begin{aligned}
& \frac{(p-2) \cdot\left[\begin{array}{l}
k(t+1)+2 \\
k(t+1)+1
\end{array}\right] q}{\left[\begin{array}{l}
k t+2 \\
k t+1
\end{array}\right]_{q}}=\frac{\left(\frac{q^{k t+2}-q^{2}}{q^{k}-1}+\frac{q-3}{2}\right) \cdot\left(q^{k}\left(q^{k t+2}-1\right)+q^{k}-1\right)}{q^{k t+2}-1} \\
= & \frac{q^{k(t+1)+2}-q^{2}-q^{k+2}+q^{2}}{q^{k}-1}+\frac{q-3}{2} \cdot q^{k}+\frac{q^{k t+2}-q^{2}+\frac{q-3}{2} \cdot\left(q^{k}-1\right)}{q^{k t+2}-1} \\
\stackrel{q=3}{=} & \frac{q^{k(t+1)+2}-q^{2}}{q^{k}-1}-q^{2}+\frac{q^{k t+2}-q^{2}}{q^{k t+2}-1} \\
<\quad & \frac{q^{k(t+1)+2}-q^{2}}{q^{k}-1}-q^{2}+1 \stackrel{q>1}{<} \frac{q^{k(t+1)+2}-q^{2}}{q^{k}-1}-\frac{q^{2}-1}{2}=m
\end{aligned}
$$

a contradiction. Thus we have $A_{3}(k(t+1)+2,2 k ; k) \leq \frac{3^{k(t+1)+2}-3^{2}}{3^{k}-1}-\frac{3^{2}+1}{2}$.

\section{CONCLUSION}

For the size of a maximum partial $k$-spread in $\mathbb{F}_{q}^{n}$ the exact formula $A_{q}(k(t+1)+r, 2 k ; k)=q^{r} \cdot \frac{q^{k(t+1)}-1}{q^{k}-1}-q^{r}+1$ was conjectured for some time, where $n=k(t+1)+r$ and $1 \leq r \leq k-1$. Codes with these parameters can easily be obtained via combining some MRD codes, see Observation 3.4. However, the conjecture is false for $q=2, k=3, n \equiv 2(\bmod 3)$, and $n \geq 8$, as we know since [6]. In this paper we have shown that the conjecture is true for $q=2, k \geq 4, n \equiv 2(\bmod k)$, and $n \geq 2 k+2$. With respect to upper bounds, Theorem 2.3 is one of the most general and sweeping theoretical tools. For the spread case, i.e., $n \equiv 0(\bmod k)$, it was sufficient to consider the (empty) set of holes. The main idea of Beutelspacher for the case $n \equiv 1(\bmod k)$, may roughly be described as the consideration of holes in the projections of partial $k$-spreads in hyperplanes. In this sense, our work is just the continuation of projecting two times ${ }^{10}$ If $k \geq 4$ the projected codewords can be distinguished from the holes by the attained dimensions. So, we naturally ask whether our result can be generalized to arbitrary $q$. In Lemma 4.6 we were able to reduce the previously best known upper bound by 1 for the special field size $q=3$. Looking closer at our arguments shows that for further progress additional ideas are needed.

In general, one may project $k-2$ times without being confronted with an interference between the projected codewords and the set of holes contained in the $(n-k+2)$-dimensional subspaces. Can this rough idea be used to obtain improved upper bounds for $r \geq 3$ and $k \geq r+2$ ?

Our main result suggest that the code attaining $A_{2}(8,6 ; 3)=34$ is somehow specific. As mentioned before, it cannot be obtained by the construction from Observation 3.4 . Even more, it cannot be obtained by the more general, so-called, Echelon-Ferrers (or multi-level) construction from [8]. So, a better understanding of the corresponding codes might be the key for possibly better constructions beating the currently best known lower bounds for e.g. $A_{2}(11,8 ; 4)$ or $A_{2}(14,10 ; 5)$.

We would like to mention a new on-line table for upper and lower bounds for subspace codes at

$$
\text { http://subspacecodes.uni-bayreuth.de, }
$$

see also [13] for a brief manual and description of the methods implemented so far. Actually, our research was initiated by looking for the smallest set of parameters, in the binary partial spread case, where the currently known lower and the upper bounds differ by exactly 1: $65 \leq A_{2}(10,8 ; 4) \leq 66$. The other cases with a difference of one are exactly those that we finally covered by Theorem 4.3 . Now, the smallest unknown maximal cardinality of a partial $k$-spread over $\mathbb{F}_{2}^{n}$ is given by $129 \leq A_{2}(11,8 ; 4) \leq 133$ and also the other cases, where the upper and the lower bound are exactly 4 apart, show an obvious pattern. At least for use, the mentioned database was very valuable. As it commonly happens that formerly known results were rediscovered by different authors, we would appreciate any comments on existing results, that are not yet included in the database, very much.

\footnotetext{
${ }^{10}$ The specific use of Theorem 3.5 is just a shortcut, resting on the same rough idea. However, it points to an area where even more theoretic results are available, that possibly can be used in more involved cases.
} 
Partial $k$-spreads have applications in the construction of orthogonal arrays and $(\bar{s}, \bar{r}, \mu)$-nets ${ }^{11}$, see [4]. Thus, Theorem 4.3 also implies restrictions for these objects. The derivation of the explicit corollaries goes along the same lines as presented in [6].

\section{REFERENCES}

[1] J. André, Über nicht-desarguessche Ebenen mit transitiver Translationsgruppe, Mathematische Zeitschrift 60 (1954), no. 1, 156-186

[2] A. Beutelspacher, Partial spreads in finite projective spaces and partial designs, Mathematische Zeitschrift 145 (1975), no. 3, 211229.

[3] P. Dembowski, Finite Geometries: Reprint of the 1968 edition, Springer Science \& Business Media, 2012.

[4] D.A. Drake and J.W. Freeman, Partial t-spreads and group constructible ( $s, r, \mu)$-nets, Journal of Geometry 13 (1979), no. 2, 210 216.

[5] J. Eisfeld and L. Storme, $t$-spreads and minimal t-covers in finite projective spaces, Lecture notes, Universiteit Gent, 29 pages (2000).

[6] S. El-Zanati, H. Jordon, G. Seelinger, P. Sissokho, and L. Spence, The maximum size of a partial 3-spread in a finite vector space over GF(2), Designs, Codes and Cryptography 54 (2010), no. 2, 101-107.

[7] T. Etzion, Problems on q-analogs in coding theory, arXiv preprint: 1305.6126, 37 pages (2013).

[8] T. Etzion and N. Silberstein, Error-correcting codes in projective spaces via rank-metric codes and Ferrers diagrams, IEEE Transactions on Information Theory 55 (2009), no. 7, 2909-2919.

[9] T. Etzion and L. Storme, Galois geometries and coding theory, Designs, Codes and Cryptography (to appear), 49p.

[10] E.M. Gabidulin, Theory of codes with maximum rank distance, Problemy Peredachi Informatsii 21 (1985), no. 1, 3-16.

[11] O. Heden, On the length of the tail of a vector space partition, Discrete Mathematics 309 (2009), no. 21, 6169-6180.

[12] O. Heden, J. Lehmann, E. Năstase, and P. Sissokho, The supertail of a subspace partition, Designs, Codes and Cryptography 69 (2013), no. 3, 305-316.

[13] D. Heinlein, M. Kiermaier, S.Kurz, and A. Wassermann, Tables of subspacecodes, University of Bayreuth, 2015, available at http://subspacecodes.uni-bayreuth.de.

[14] S.J. Hong and A.M. Patel, A general class of maximal codes for computer applications, IEEE Transactions on Computers 100 (1972), no. $12,1322-1331$

[15] D. Silva, F.R. Kschischang, and R. Koetter, A rank-metric approach to error control in random network coding, IEEE Transactions on Information Theory 54 (2008), no. 9, 3951-3967.

[16] V. Skachek, Recursive code construction for random networks, IEEE transactions on Information Theory 56 (2010), no. 3, 1378-1382.

Department of Mathematics, University of BAyreuth, 95440 BAyreuth, Germany

E-mail address: sascha.kurz@uni-bayreuth.de

${ }^{11}$ Using the notation from this paper, we have $\bar{s}=q^{k}, \bar{r}=A_{q}(n, 2 k ; k)$, and $\mu=q^{n-2 k}$. 